\title{
Design and Implementation of Travelling Wave Electrode Silicon Mach Zehnder Modulator based Plus-Shaped PN Junction Phase Shifter for Data Centre Application
}

\author{
R. G. Jesuwanth Sugesh ${ }^{1 *}$, A. Sivasubramanian ${ }^{2}$ \\ ${ }^{1 *}$ Research Scholar, Vellore Institute of Technology, School of Electronics Engineering, Chennai, \\ India. \\ ${ }^{2}$ Vellore Institute of Technology, School of Electronics Engineering, Chennai, India.
}

Received: January 5, 2021. Revised: June 29, 2021. Accepted: July 12, 2021. Published: July 15, 2021.

\begin{abstract}
Silicon Mach-Zehnder modulator (SMZM) is fit to high-contrast optical modulation in wide ranges of spectral along 50 Gbaud symbol rates. Some times its efficiency is lower in wavelength about $1.31 \mu \mathrm{m}$ than 1.55 $\mu \mathrm{m}$, it can decrease the Phase Shifter (PS) efficiency and occupies large amount of data rates. In this manuscript, the plus-shaped PN junction Phase Shifter (PS) is proposed. The major aim of the proposed work is to create an optimum CD type plus-shaped PS including lesser $V \pi \mathrm{L}$. Silicon MZM including proposed PS can satisfy the higher-speed data transmission requirement on the applications of inter with intra data centre. The objective of this method is to increase the modulation efficiency (ME) by decreasing the optical loss for higher-speed data rate (DR). To get greater efficiency of modulation, the $\mathbf{P}$ doped region width and the thickness of doped regions are differ under slabs. The simulation analysis of circuit-level is executed in the proposed PS acquired at travelling wave electrode (TWE) silicon Mach Zehnder modulator. In 80 Gbps, the 12.39 dB maximal extinction ratio along $8.67 \times 10-8$ bit error rate (BER) was acquired in $V \pi L \pi$ of $1.05 \mathrm{~V} . \mathrm{cm}$ for $3.5 \mathrm{~mm}$ PS length. The measured intrinsic bandwidth of $3 \mathrm{~dB}$ denotes $\sim 38 \mathrm{GHz}$, whereas the transmission energy per bit denotes $1.71 \mathrm{pJ} / \mathrm{bit}$. More examines are carried out to recognize the maximal communication distance using proposed PS under SMZM for the requirements of data centre.
\end{abstract}

Keywords-PN junction PS, SMZM, bit error rate, silicon photonic device, and data centre.

\section{INTRODUCTION}

Nowadays, a photonic devices measuring is the investigation of interest for satisfying the development of dangerous requirements at data centres. The phase shifter (PS) performance is computed the modulator performance. Technological advances, like 5G, Internet of Things (IoT), Artificial Intelligence (AI) have leaded the way for increasing internet traffic (IT). IT is developed in alarm rate owing to file sharing enhancement, video calls and streaming, online conferences etc $[1,2]$. This increasing IT has force on data centres to implement such demands. The union of international telecommunication has issued procedures governing the use of optical bandwidth. The technology of Silicon photonics $(\mathrm{SiPh})$ is suggested to fulfil the requirement of futuristic network. SiPh offers a cost-effectual mode for incorporating the electronic with photonic modules at Si chip by the benefits of CMOS fabrication technology [3] \& [4].

The optical modulator is a noteworthy part of higher-speed data transmission connection amid the electronic and optic mechanisms [5-7]. Materials along hybrid optical modulators, viz Indium Tin Oxide [ 8 -14] offer maximal proficiency of modulation, but it is not compatible CMOS fabrication, like silicon. At silicon optical modulators, the modulation imitates the major plasma scattering effect. In the doped region, a carrier concentration is caused by external voltage bias that leads to effectual difference of index $[15,16]$. The carrier injection (CI) and carrier depletion (CD) are occurred through forward and reverse bias voltage respectively, which is utilized the standard plasma scattering strategies. Lesser $3 \mathrm{~dB}$ bandwidth is a major drawback of CI strategy, because maximal capacity of dispersion junction, long lifespan of freecarrier, acquired maximal ME (low V $\pi \mathrm{L}$ ) [4]. The CD strategy handles this shortcoming, also supports higher-speed data 
process [17] \& [18]. The drawback of CD is less ME (high $\mathrm{V} \pi \mathrm{L})$. The obtainable MZM is chosen to its thermal steadiness, simple fabrication, with higher efficiency amid those optical modulators. Consuming maximal power with huge footprint is the drawback of MZM [19, 20].

The efficiency of modulator depends upon the effective of PS utilizing doping pattern and concentration. By utilizing doping pattern, the PS is categorized as interleaved, horizontal, vertical [21 -29]. Multi-PN junction including PS length presents greater modulation proficiency at interleaved type PS but infabrication complexity cost. Horizontal type PS reduced the fabrication complexity, and then lesser doping concentration is employed in PS length cost. The PS length is lessened in vertical type depending on high doping concentrated vertical slabs. Along the carrier concentration increment, increases the free carrier absorption loss. Slot like structures is employed [30 -34] to minimize the loss, but maximize the interaction of light-object. TWE is selected on combined structure, owing to its independent of RC time stable. It helps $\mathrm{CD}$ technique [33 -35] for getting huge footprint.

The presented report includes workflow model for biomedical data. The proposed model is a solution for the case study of two-dimensional data classification. The experiments are conducted using distance computing techniques: Euclidean distance, Manhattan Distance, Cosine Distance. The result values show the percentage of accurately classified images for the three distance measures. In addition, the paper also presents a workflow model as a solution for prediction problems. The experiments are performed to compute the efficiency and probability of heart disease occurrence using Logistic Regression, Decision Tree, and Random Forest with Naïve Bayes techniques. Results show the advantages and disadvantages of the included methods [36]. Multidimensional tries hashing (MTH) access method is an extension of the tries hashing for dynamic multi-key files (or databases). Its formulation consists of maintaining in main memory (d) separate tries, and then every index has an attribute. The data file represents an array of dimension (d), in an order, linear way on the disk. The relationship amid the physical addresses and indexes resulting of the application of the tries is achieved through the mapping function. In average, a record may be found in one disk access, which places the method among the most efficient known. Yet MTH has the double disadvantage of a low occupancy of file buckets (40-50\%) and a greater memory space in relation to the file size (tries in memory) [37].

The transfer functions linearity of ME-MZM along PN junction PS has been examined. Here, experimentally proves $168 \mathrm{~Gb} / \mathrm{s}$ 4-level pulse amplitude modulation signals (PAM-4) generation utilizing multi-electrode Mach-Zehnder modulator (ME-MZM) driven via 2 on-off keying (OOK) electrical signals, it is trained by lesser digital signal processing (DSP) that involves pulse shaping in symbol rates for 84 Gbaud, RF spectral pre-compensation filter, clipping including quantization. Here, the transmission presentation of ME-MZM is likened with typical SiP SPP travelling wave Mach-Zehnder modulator (TWMZM) [38] utilizing different fiber lengths. It permits to quantitatively liken PAM-4 signal production at optical domain attained through ME-MZM Vs TWMZM that necessitates PAM-4 signal as created in electric domain. Moreover, the enhancement of transmission proficiency is attained through DSP. Here, report $128 \mathrm{~Gb} / \mathrm{s}$ PAM-4 effectual transmissions over $2 \mathrm{~km}$ of fiber below the KP4 forward Error correction (FEC) threshold of $2.0 \times 10-4$ at C-band. MEMZM is reached maximal bit rate transmission owing to high bandwidth of every sector likened to related TWMZM. The experimental shows ME-MZM functionality based that needs on-off keying drive signals, maximal baud PAM-4 generation is reached. Every modulator provides the power consume per bit for different bitrates with the scheme of modulation. When likened to TWMZM, this experimental outcome demonstrates that the ME-MZM gives effectual solution for maximal baud PAM-4 Silicon MZMs are fit to higher-contrast optical modulation at broad spectral ranges along 50 Gbaud high symbol rates. Some times its efficiency is lowers in wavelengths about $1.31 \mu \mathrm{m}$ than $1.55 \mu \mathrm{m}$, this will decrease the PS efficiency and occupies large amount of data rates. To overcome this problem this paper is proposed. Through diminishing the doping region, the merits of horizontal with vertical mode doping is utilized at PS. Therefore, in this manuscript, a plus-shaped PN junction PS models along higher ME is proposed. The aim is to create an optimum CD type plus-shaped PS including lesser V $\pi \mathrm{L}$. Silicon MZM including proposed PS can satisfy the higher-speed data transmission requirement on the applications of inter with intra data centre.

The contribution of this paper is summarized below:

In this manuscript, the plus-shaped PN junction PS is deemed. The aim is to create an optimum CD type plus-shaped PS including lesser V $\pi \mathrm{L}$.

Silicon MZM including proposed PS can satisfy the higherspeed data transmission requirement on the applications of inter with intra data centre.

The objective of this method is to increase the modulation efficiency (ME) by decreasing the optical loss for higherspeed data rate (DR).

To get greater efficiency of modulation, the $\mathrm{P}$ doped region width and the thickness of doped regions are differ under slabs.

The remaining of this manuscript explains section 2 as Literature survey, the device structure is delineated in segment 
III. The experimental outcomes have been assessed in segment IV.

\section{LITERATURE SURVEY}

Wang et al. [39] have presented the Linearity properties of double-parallel silicon MZM. Here, maximal linearity wideband double-parallel silicon MZM. The presented modulator contains 2 carrier-depletion-depend MZMs along single-drive push-pull traveling wave electrode design. The analytic mode was designed for examining the modulator's linearity property. The outcomes display that the modulator could successfully suppress third-order harmonics with the distortion of inter-modulation, upgraded modulation linearity likened to single MZM.

Veera Subramanian, et al. [40] have presented a silicon photonic double-parallel MZM working near $1550 \mathrm{~nm}$ utilized to PAM-4. Here, differential together with integral nonlinearity was assessed. Driving as well as biasing mode enhances the linearity with PAM function. The computed $2^{\text {nd }}$ harmonic distortion with two-tone, $3^{\text {rd }}$-order inter-modulation spurious-free dynamic range were $75 \mathrm{~dB} \cdot \mathrm{Hz}^{1 / 2}, 86 \mathrm{~dB}$. $\mathrm{Hz}^{3 / 2}$. Moreover, the device proficiency was examined in a short-attain transmission scheme.

Ghilioni et al. [41] have presented modulator design depending on Mach-Zehnder for the recognition of higher DR Silicon Photonics transmitters. Here, the challenges posed by presented devices incorporation on silicon were assessed. Here, multiple stages, travelling wave, were focuses on power capacity. A significant parameter assuming the stringent necessities of standard module form factors. A 25 Gbps multiple stages together with 56 Gbps travelling wave modulator were deemed. Every electro-optical transmitter was acquired through 3D assembly of electronic IC on top of photonic IC with the help of copper pillars. 30\% optimum efficiency of power likened to Silicon Photonics state-of-theart in same DRs as well as the performance of comparable extinction ratio was proved in both cases. Since packaging was an important component of Silicon Photonics higher volume creation, packaged chips were reported.

Baudot et al. [42] have presented a lesser voltage $25 \mathrm{Gbps}$ silicon MZM at O-band. Here, a silicon modulator depending upon symmetric Mach-Zehnder interferometer operating at optical communication schemes O-Band. Even though the effect of FCPD was inefficient in wavelengths $1.31 \mu \mathrm{m}$ than $1.55 \mu \mathrm{m}$, the presented modulator shows an ME likened with state-of-the-art counterparts at C-Band, $\mathrm{V}_{\pi} \mathrm{L}_{\pi}$ product amid $0.95 \mathrm{~V} \mathrm{~cm}$ and $1.15 \mathrm{~V} \mathrm{~cm}$. It was feasible to prove higher speed mode in $25 \mathrm{~Gb}$ ps utilizing driving voltage as lesser 3.3 $\mathrm{V}_{\mathrm{pp}}$, compatible to the technology of BiCMOS.

Shao et al. [43] have presented a model for improving silicon Mach-Zehnder optical modulator linearity with the help of doping control. Enhance the silicon carrier-depletion Mach-Zehnder optical modulator linearity presentation by regulating the doping concentration. In waveguide, optical field distribution was Gaussian-like distribution. If maximize the concentration of doping, then minimize the dynamic depletion width of PN junction under the same modulation signal, also the overlap incorporation width amid the Gaussian-like optical field distribution with depletion region was lesser. So, the modulated signal contains lesser nonlinear modules. Here, various devices were fabricated along various doping concentrations. By adopting a ten times doping concentration, the spurious free dynamic range (SFDR) for third-order inter-modulation distortion maximizes 109.2 dB.Hz $\mathrm{Hz}^{2 / 3}$ to $113.7 \mathrm{~dB} . \mathrm{Hz}^{2 / 3}$, also SFDR for second harmonic distortion (SHD) maximizes $87.6 \mathrm{~dB} \cdot \mathrm{Hz}^{1 / 2}$ to $97.5 \mathrm{~dB} . \mathrm{Hz}^{1 / 2}$ in $2 \mathrm{GHz}$ driving frequency.

A. Comparative Analysis

\begin{tabular}{|c|c|c|c|}
\hline Methods and Authors & Objective & Experimental results & Dis advantages \\
\hline $\begin{array}{l}\text { Wang et al. [35] have presented the } \\
\text { Linearity characterization of dual- } \\
\text { parallel silicon MZM. In this higher } \\
\text { linearity wideband dual-parallel } \\
\text { silicon MZM. }\end{array}$ & $\begin{array}{l}\text { The presented modulator } \\
\text { contains } 2 \text { carrier-depletion- } \\
\text { depend MZMs along single- } \\
\text { drive push-pull traveling } \\
\text { wave electrode configuration. } \\
\text { Analytic mode was } \\
\text { implemented to examine the } \\
\text { linearity property of } \\
\text { modulator. }\end{array}$ & $\begin{array}{l}\text { The measurement } \\
\text { outcomes display that the } \\
\text { modulator could } \\
\text { successfully suppress third- } \\
\text { order harmonics, } \\
\text { distortions of third-order } \\
\text { inter-modulation, upgraded } \\
\text { modulation linearity, } \\
\text { compared to a single } \\
\text { MZM. }\end{array}$ & $\begin{array}{l}\text { Lesser } 3 \mathrm{~dB} \text { bandwidth is a } \\
\text { major drawback of } \mathrm{CI} \\
\text { strategy, because maximal } \\
\text { capacity of dispersion } \\
\text { junction, long lifespan of } \\
\text { free-carrier, acquired } \\
\text { maximal ME (low } \mathrm{V} \pi \mathrm{L} \text { ) }\end{array}$ \\
\hline $\begin{array}{l}\text { Veerasubramanian et al. [36] have } \\
\text { presented a present a silicon } \\
\text { photonic double-parallel MZM } \\
\text { functioning } 1550 \mathrm{~nm} \text { for PAM- } 4 \text {. }\end{array}$ & $\begin{array}{c}\text { A driving scheme and a } \\
\text { biasing method that improves } \\
\text { the linearity as well as PAM } \\
\text { operation of device }\end{array}$ & $\begin{array}{l}\text { The measured second } \\
\text { harmonic distortion, two- } \\
\text { tone, } 3^{\text {rd }} \text {-order inter- } \\
\text { modulation spurious-free }\end{array}$ & $\begin{array}{l}\text { Consuming maximal } \\
\text { power with huge footprint } \\
\text { is the drawback of MZM }\end{array}$ \\
\hline
\end{tabular}


Ghilioni et al. [37] have presented modulator schemes depending on Mach-Zehnder for the recognition of higher-DR Silicon Photonics transmitters.

Baudot et al. [38] have presented Lesser voltage $25 \mathrm{Gbps}$ silicon MZM in O-band.

Shao et al. [39] have presented a model for improving the silicon Mach-Zehnder optical modulator linearity utilizing doping control multistage and travelling wave methods were used to increase the power efficiency

silicon modulator depending upon symmetric MachZehnder interferometer operating at O-Band of optical communication schemes

Enhance the linearity performance of silicon carrier-depletion MachZehnder optical modulator by regulating doping concentration. dynamic range $75 \mathrm{~dB} \cdot$

$\mathrm{Hz}^{1 / 2}, 86 \mathrm{~dB} \cdot \mathrm{Hz}^{3 / 2}$.

$30 \%$ optimum power

capacity likened to Silicon

Photonics state-of-the-art

in same DR, also

comparable extinction ratio

performance was

demonstrated in both cases.

Demonstrate higher speed function on $25 \mathrm{~Gb}$ ps utilizing driving voltage lower $3.3 \mathrm{~V}_{\mathrm{pp}}$, compatible with BiCMOS technology.

Performances of the speed

Experimental results indicate that SFDR for $3^{\text {rd }}$ order inter-modulation deformation maximizes 109.2 dB. Hz ${ }^{2 / 3}$ to 113.7 $\mathrm{dB} . \mathrm{Hz}^{2 / 3}$ and SFDR for $2^{\text {nd }}$ harmonic distortion maximizes $87.6 \mathrm{~dB} \cdot \mathrm{Hz}^{1 / 2}$ to $97.5 \mathrm{~dB} . \mathrm{Hz}^{1 / 2}$ in $2 \mathrm{GHz}$ driving frequency through adopting ten times doping concentration. was low

the modulation imitates the major plasma scattering effect

Consumes large number of power.

\section{DEVICE STRUCTURE}

Rib waveguide and $500 \mathrm{~nm}$ width $\left(\mathrm{W}_{\text {rib }}\right)$, thick of ridge $220 \mathrm{~nm}$ ( $\left.\mathrm{t}_{\mathrm{rib}}\right), 90 \mathrm{~nm}$ etching deepness has been deemed because it provides good optical confinement along the functioning of single-mode $\left(\mathrm{TE}_{1}\right)$, also this is a standards of fabrication.

The simulation is carried out in analytical mode [37]. Figure 1 depicts the light propagation via rib waveguide. From the waveguide centre $100 \mathrm{~nm}$ offset is imitated to PN junction for improving the efficiency of phase. $\mathrm{N}$ doped region width set as $150 \mathrm{~nm}$, slab thickness $(\mathrm{P}, \mathrm{N})$ set $70 \mathrm{~nm}$. The concentrations of $\mathrm{P}$ as well as $\mathrm{N}$ doped set $9 \times 10^{17} \mathrm{~cm}^{-3}$ with $7 \times 10^{17} \mathrm{~cm}^{-3}$, because holes contain huge shift index with lesser absorb than electrons [7]. At the edge of waveguide, slab resistance has been diminished to $1 \times 10^{19} \mathrm{~cm}^{-3}$ carrier concentration of $\mathrm{P}+$ together with $\mathrm{N}+$ doped region. Aluminium electrodes (Al) have been employed to electrical contacts. The PS (L) length is set as $3.5 \mathrm{~mm}$. Figure 2 depicts 3 dimensional view of plusshaped PS. Maximal $5 \mathrm{~V}$ driving voltage is utilized to cathode $(\mathrm{V}=0$ to $5 \mathrm{~V}$ ) for maintaining minimal power consumption. Silicon MZM has lateral PN-junction rib-waveguide PS that is portrayed in Figure 2. Si MZM contains single MZ interferometer as well as RF coplanar waveguide in MZI. Lateral PN junction is produced at $\mathrm{Si}$ rib-waveguide PS including $\ell=3$ or $4 \mathrm{~mm}$ on both MZI. The rib waveguide is structured to the dimension of rib width $500-\mathrm{nm}$, rib height 220-nm, side slab height $95-\mathrm{nm}$ for optical modulation at single mode transverse-electric polarization. The dimensions are deemed to attain maximal speed of modulation with minimal optical loss. Maximal slab presents lesser resistance series with rapid phase modulation, while optical loss implies greater owing to weak mode confinement at middle rib along slab height $<95 \mathrm{~nm}$. Si MZMs fabricates 8-inch SOI wafers of $220-\mathrm{nm}$ SOI thickness together with $2-\mu \mathrm{m}$ buried-oxide (BOX) utilizing 130-nm node CMOS. BOX layer helps the bottom clad. In every PS, DC reverse-bias voltage has been employed to $\mathrm{PN}$-junction for maximal speed refractive-index modulation in the mode of carrier-depletion.

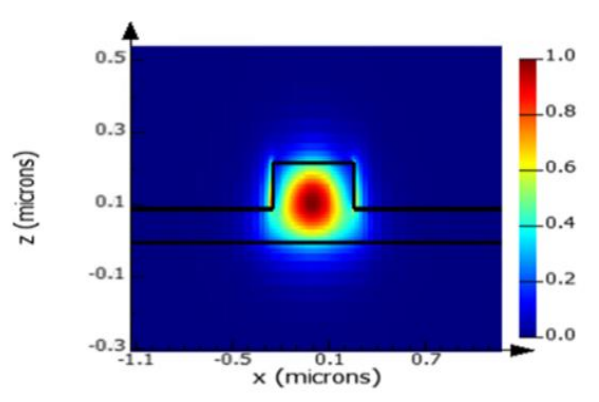


Figure 1: propagation of lightvia rib waveguide $(500 \times 220 \mathrm{~nm})$

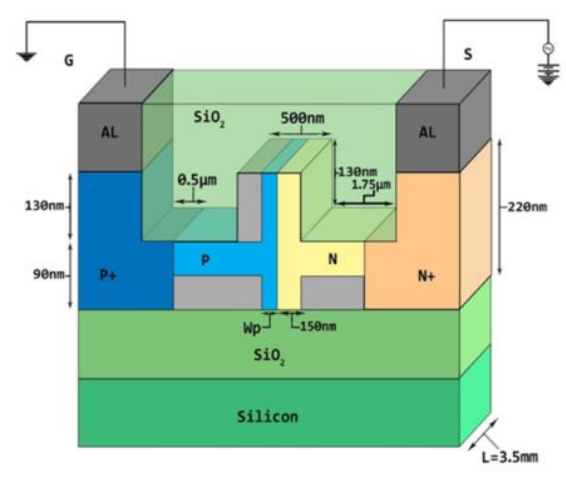

Figure 2: (3 dimensional view of plus-shaped PS)

Capacitive combination of reverse-biased PN junction is involved radio frequency CPW simulation for obtaining 10$\mu \mathrm{m}$ signal electrode width as well as gaps $6.4-\mu \mathrm{m}$ width gaps amid the signal and ground electrodes for $50-\Omega$ matching impedance. RF electrodes are covered by $\mathrm{Al}$ metal in $2-\mu \mathrm{m}$ thick top clad with the thickness of $2-\mu \mathrm{m}$. The signal electrode is linked with rib waveguide P-doped slab, a ground electrodes for rib waveguide $\mathrm{N}$-doped slab utilizing $\mathrm{Al}$ vertical interlink access. Signal electrodes are in top of PS, then linked to contact pads removed in both sides. Figure 2 depicts the 2 carrier-depletion Si MZMs profile has lateral PN-junction in rib-waveguide PS for DC FOM evaluation. Rib-waveguide PS contains specific profile of PN-junction, here left half of silicon core at rib waveguide is uniformly doped with P-type dopants (boron) and right half of the core is uniformly doped with N-type dopants. Depletion region forms at $\mathrm{PN}$-junction vertical boundary in the rib core center. The compensation doping permit optical loss minimization at PN-junction PS without maximizing $\pi$-shift voltage $(\mathrm{V} \pi)$.

\section{RESULT ANALYSIS}

The analysis is categorized as 2 level simulation: (i) device (ii) system (Figure 3). On device-state, the electro-optic as well as RF behaviour of PS is carried out with the help of finite-difference eigenmode (FDE) evaluation (Figure 3.a). In system-level, the PS parameters design is derived from MZM (PS-MZM) in Lumerical Interlink (Figure 3.b). The longdistance transmission as well as higher-speed DR is implemented for examining the proficiency of PS-MZM's to the application of data centre on and off-chip transmission. The parameter explanation of figure $3 \mathrm{~b}$ is given below:

1. Pseudo-random bit sequence (PRBS) generator:

Typical BERT and PRBS generator have been structured to the technology of silicon bipolar or SiGe BiCMOS [1][2]. Besides, a standard PRB series contains ratio of mark density that is measured by count of zeros average ratio to 112 series. For simulating the DC unbalance code test vehicle, mark densities rather than half, viz U4 or 118 have been desirable. The PRBS core as well as mark density control is injected for the generator of mark density. At common PRB series, " 0 and "1" probabilities = half, then PRB series is non-correlated with delay version. Here, 2 adjacent hits are ended together, "I" shows in output while both bits imply 1 , and then probability of "I" is 114 . Thus, the PRBS of mark density 118 is reached by "ending" the neighboring 3 bits.

\section{Essential Signal generator and Modulator:}

Digital signals have been modulated utilizing DSP in its phase together with the components of quadrature phase. These digital signals converts analogue, then modulated as $900 \mathrm{MHz}$ frequency band with the help of frequency hop local oscillator. Data is transferred at r.f. power bursts along bursts amplitude profile as mentioned by photo detector.

\section{Photo detector}

Photo detector is a device that is used to convert photons to electrons. These detectors depend upon photons absorption rate rather than energy absorption rate not like the thermal detectors. Nevertheless, a device could absorb photons only if the energy of incident photons is above some minimal threshold.

\section{BER Tester}

Bit Error Rate (BER) Tester is utilized for determining the count of errors at data transmission process. Data transfer is any part of the device that is considered in the data and modifies the data.

\section{Eye diagram analyzer and the Lazar}

The 3 dimension EO transmit including measurements of eye diagram is determined through 231-1 PRBS from sample generator in EIC input as well as optical output is captured utilizing digital communication analyzer. Erbium doped fiber amplifier (EDFA) along tunable bandpass filter $(0.8 \mathrm{~nm}$ bandwidth) has been utilized to optical amplify the optical output signal from chip. For Si MZM, the input wavelength is set to device quadrature point. 


\section{Device level simulation}

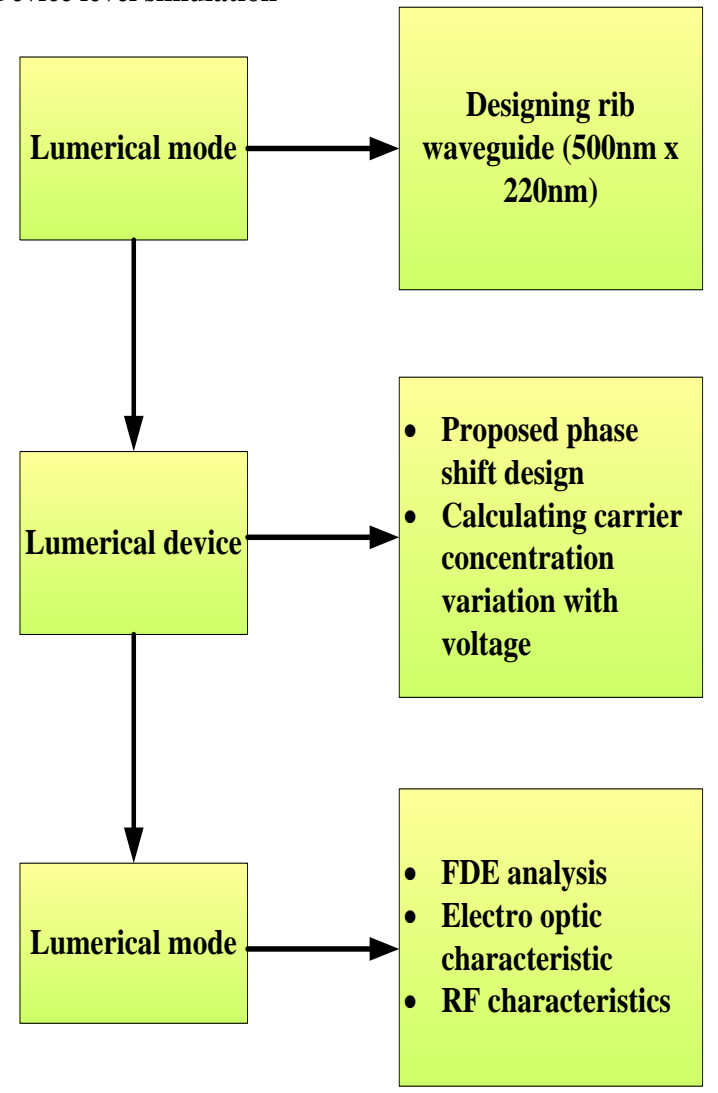

Figure 3: Simulation flow for result analysis (a) Device-level simulation

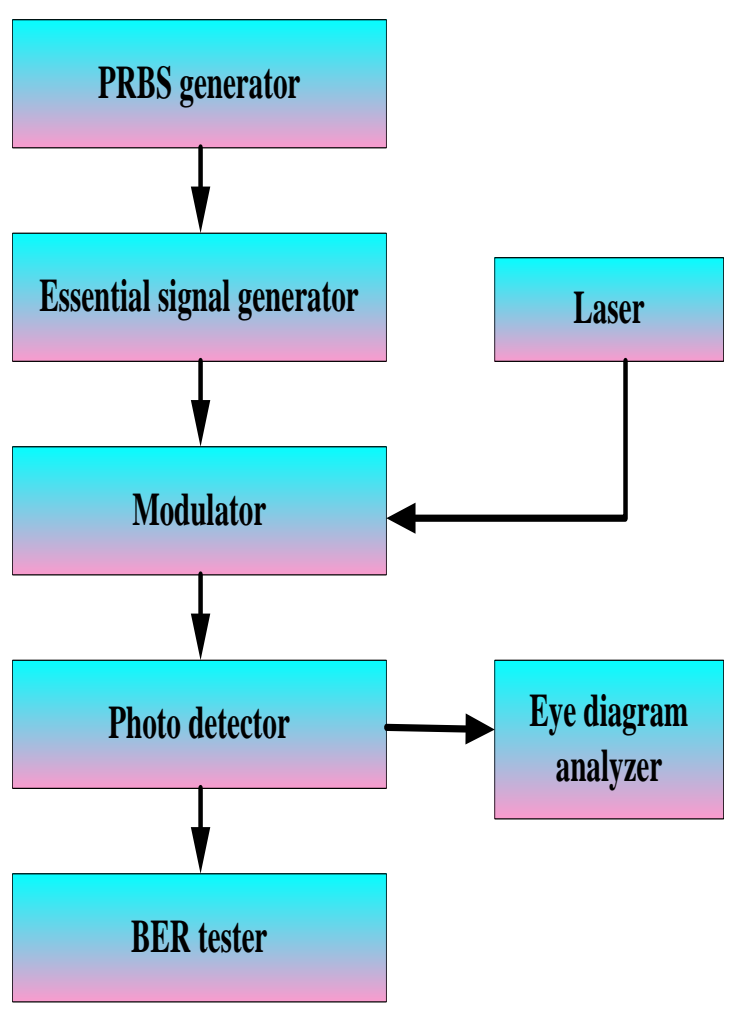

Figure 3b: Simulation flow for result analysis (b) System-level simulation

\section{A. Assessment of Device level}

The reverse bias voltage (BV) of PS is varies for analysing the electro-optic PS performance. When BVis maximized, the carriers have been depletion from PN junction. It diminishes the density of carrier, so diminished the junction capacitance that is represented at Figure 4. The junction capacitance $(\mathrm{C})$ depending upon electron $\left(N_{n}\right)$, density (1), hole $\left(N_{p}\right)$.

$C=\frac{t_{r i b} \times \sqrt{ }\left(q \varepsilon_{0} \varepsilon_{r}\right)}{\left.\sqrt{\left(2\left(N_{p}-1+N_{M}-1\right)\right.} \times\left(V_{v}-V\right)\right)}$

Here, q implicates electric charge, $\varepsilon_{0}$ specifies dielectric constant, $\varepsilon_{r}$ as relative permittivity, $V_{v}$ as dispersion capacity.

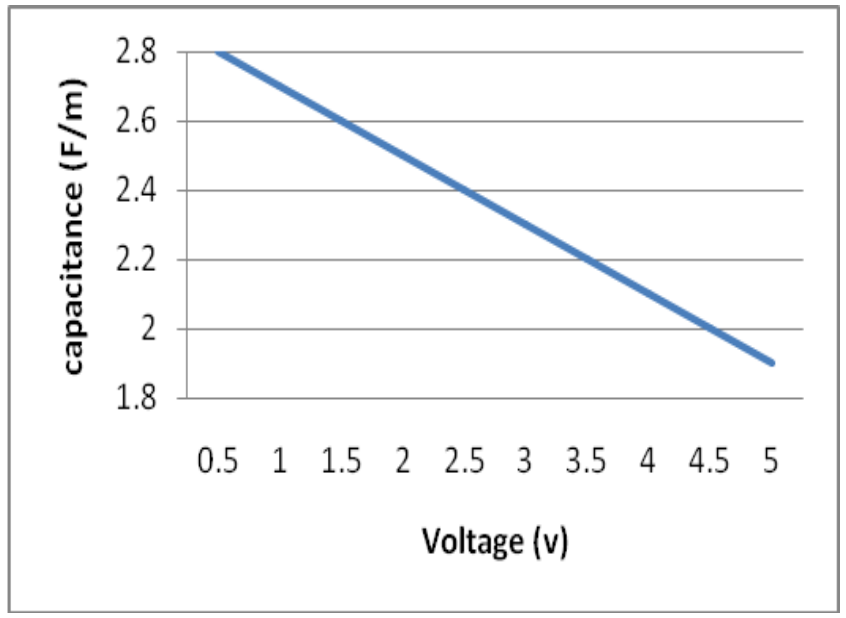

Figure 4: Junction Capacitance ofPS for varying BV

$\mathrm{CD}$ is the impact of refractive index $(\Delta \mathrm{n})$, absorption coefficient $(\Delta \alpha)$ of PS. The derivation (2) and (3) is exhibited by So ref and Bennett [14].

$$
\begin{aligned}
& \Delta \mathrm{n}=-8.8 \times 10^{-22} N_{n}-8.5 \times 10^{-18} N_{p}{ }^{0.8} \\
& \Delta \alpha=8.5 \times 10^{-18} N_{n}+6 \times 10^{-18} N_{p}
\end{aligned}
$$

The optical PS feature is modified by effectual index change (5) and voltage $\left(\Delta n_{\text {eff }}(V)\right)$. It occurs phase shift $(\varphi)$ at propagating optical wave (OW) including PS length $(L)$, is exhibited in (6).

$$
n_{e f f}(V)=n_{e f f i}+\int \Delta n(V) d V
$$

Here, $\mathrm{n}_{\mathrm{eff}, \mathrm{I}}$ implies effectual waveguide index absence of doping.

$$
\Delta n_{e f f}(V)=n_{e f f}(V)-n_{e f f}(0)
$$


$\varphi(V)=\frac{2 \pi \Delta n_{\text {eff }}(V)}{\lambda L}$

The absorption coefficienta with PS as ,

$\alpha(k)=\frac{\iint \Delta \alpha(V)\left|\mathbf{E}\left(x_{y} y z\right)^{2}\right| d x d y}{\iint\left|\mathrm{E}\left(x_{y} y z\right)^{2}\right| d x d y}$

Here, $\mathrm{x}, \mathrm{y}$ implies coordinates of waveguide dimension, $\mathrm{z}$ specifies PS length coordinate, $\mathrm{E}(\mathrm{x}, \mathrm{y}, \mathrm{z})$ signifies waveguide mode optical supply of intensity.

The absorption loss (AL) is the noteworthy part of overall loss in PS. Owing to the photons absorption via carriers, the $\mathrm{AL}$ is occurred (free carrier loss of absorption). As the voltage increases, the carriers drop from the junction, it lessens the carrier AL with PS. (Figure 5). The free carriers are higher while $\mathrm{P}$ doped region ( $\mathrm{Wp}$ ) is huge, so the loss is higher via $\mathrm{PS}$ is acquired in lesser voltages. If the region of $\mathrm{P}$ doped is tiny, then decreases the loss. The necessary PS derived in maximal voltage owing to lesser variation of carrier concentration. It derives $\mathrm{Wp}=100 \mathrm{~nm}$ minimal loss, then needed $\pi \mathrm{PS}$ is acquired within the limits of voltage set. If diminishing $\mathrm{Wp}<100 \mathrm{~nm}$, then the loss diminishes in the cost of phase efficient. The necessary PS modulation is derived in $3.05 \mathrm{~V}(\mathrm{~V} \pi)$ reverse bias voltage at presented structure along $4.1 \mathrm{~dB}$ loss. Figure 6 depicts the $\mathrm{V} \pi$ difference including loss of PS length operation. $\mathrm{V} \pi$ lessens the propagation $\mathrm{OW}$ has visible for change the Phase shift of entire PS length with maximizing length. If maximizing the carriers communication, optical mode, length, then maximizes the loss, length, but minimizes the speed of operation. It creates trade-off stage amid theV $\pi$ and PS distance.

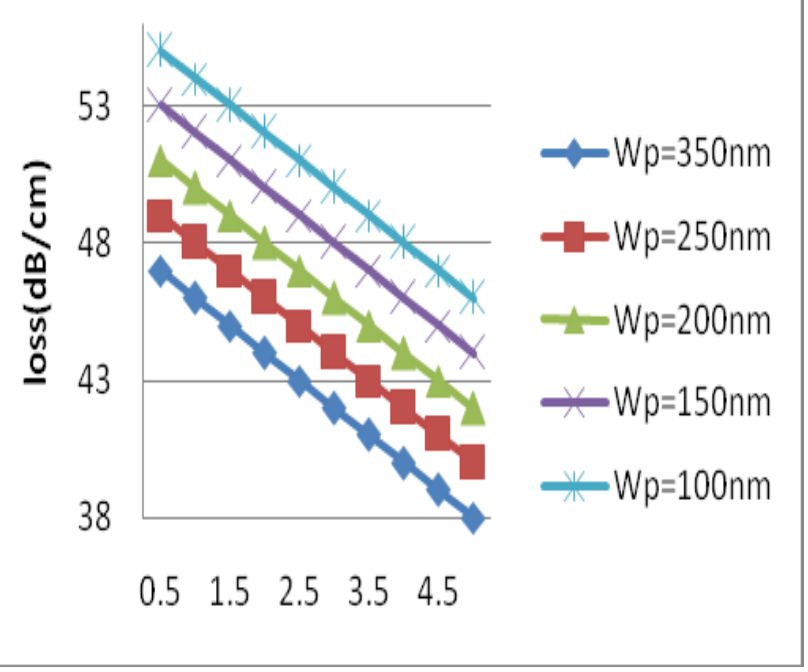

Fig 5: Loss Vs voltage to vary $\mathrm{Wp}$ at the presented design

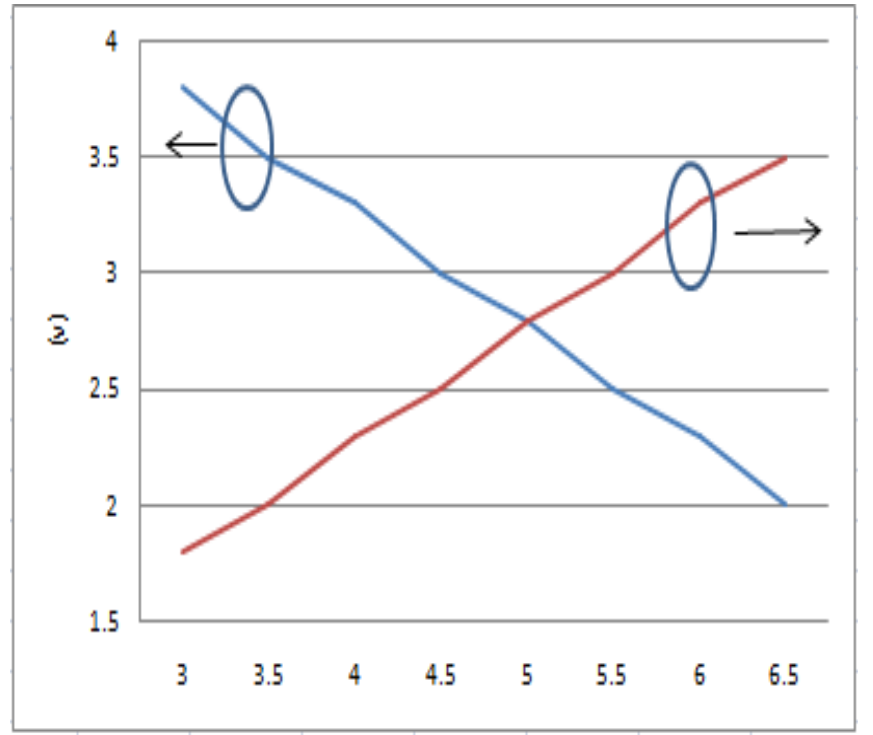

Figure 6: V $\pi$ including loss variation depending onPS length for proposed PS design along $\mathrm{Wp}=100 \mathrm{~nm}$

The $3 \mathrm{~dB}$ intrinsic bandwidth $\left(f_{3 d B}=\frac{1}{2 \pi R C}\right)$ of PS is determined as $37.7 \mathrm{GHz}$. Here, TWE is suggested for dealing the stable RC time, and then strongly connect the RF microwave including OW. To robust connect; optical group index (OGI) must be equivalent to effectual index in specific frequency. In $26 \mathrm{GHz}$, assume the index match, it leads to maximal link amid the mode of RF and optical. Figure 7 portrays the loss of $3 \mathrm{~dB} / \mathrm{cm}$ determines in $26 \mathrm{GHz}$. Here, loss is directly proportional to the frequency of microwave. To $3.5 \mathrm{~mm}$ length design, $6 \mathrm{~dB}$ bandwidth of $24.75 \mathrm{GHz}$ is reached (Figure 8), also assures that the device is deemed to the communication of higher-speed DR.

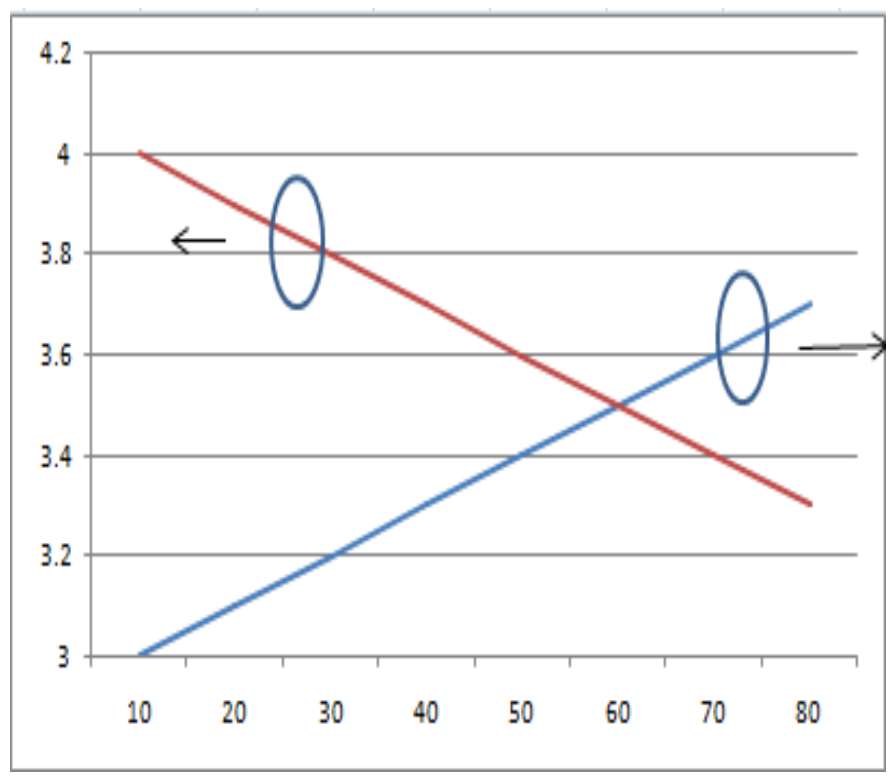

Figure 7: RF effectual index with Loss 


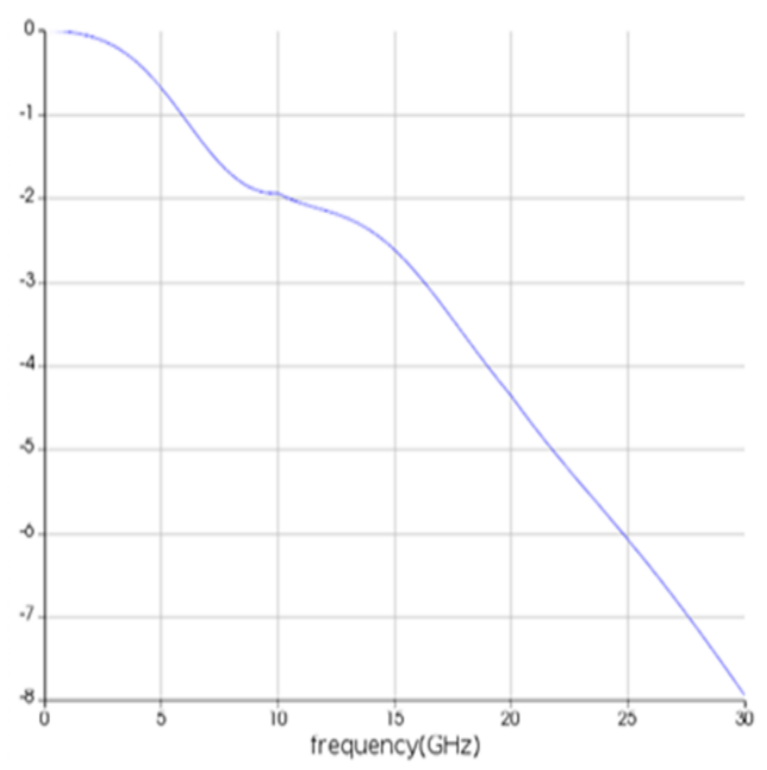

Figure 8: Electric S21 (dB) utilizing frequency

\section{B. System-level performance}

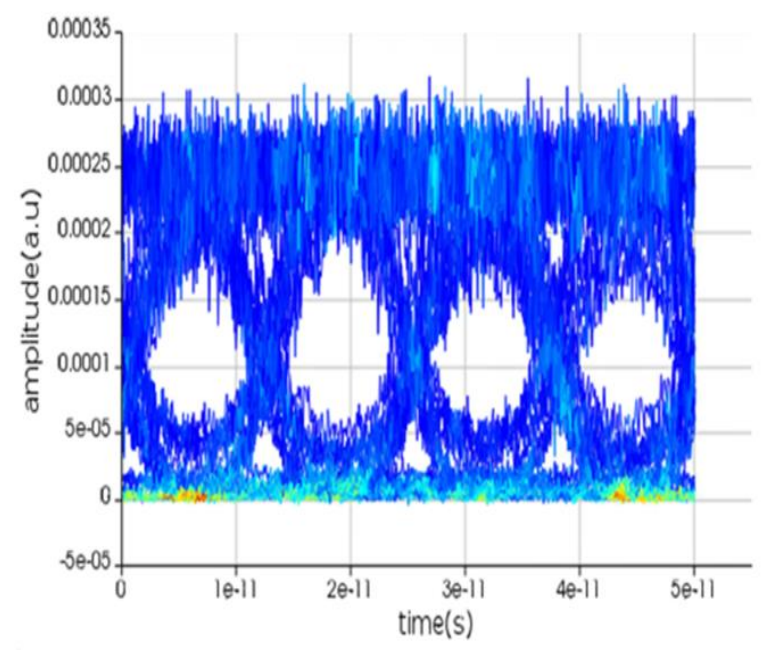

Figure 9: PS-MZM eye diagram and $\mathrm{V} \pi \mathrm{L}=1.05 \mathrm{~V} . \mathrm{cm}$

Here, the dynamic performance imbalanced MZM combined to PS design is described. PS-MZM contains $100 \mu \mathrm{m}$ deliberate length dissimilarity including long arm, which provides $0.5 \mathrm{~V}$ fixed bias voltage as well as irregular voltage swing $\left(\mathrm{V}_{\mathrm{pp}}\right)$ and direct current reverse bias $\left(\mathrm{V}_{\mathrm{dc}}\right)$ are employed to another arm. At $80 \mathrm{Gbps}$, the generator of pseudo-random bit sequence (PRBS) creates a communication data. The electrical signal producer and NRZ line coding method have been utilized for converting logical data as electric signal message. The $1552.5 \mathrm{~nm}$ carrier signal is created by CW Laser. Demodulate the acquired modulated signal utilizing photo detector responsively $1 \mathrm{~A} / \mathrm{W}$. BER including eye diagram is assess the signal of demodulated. Here, $12.39 \mathrm{~dB}$ for eye diagram together with extinction ratio (ER), $8.67 \times 10^{-8}$ for BER, $1.05 \mathrm{~V} . \mathrm{cm}$ for $\mathrm{V} \pi \mathrm{L}$ attains $3 \mathrm{~V}_{\mathrm{pp}}, 1.5 \mathrm{~V}_{\mathrm{dc}}$. The eyecrossing is approximately $50 \%$ broad eye opening guides to the deviation of minimal duty cycle with minimal inter-code interfering. It assures the PS-MZM is appropriate for higherspeed DR utilizations. The usage of energy per bit $\left(E_{\text {bit }}=\frac{C V^{2}}{4}\right)$ to data transfer is determined as $1.71 \mathrm{pJ} / \mathrm{bit}$. The proficiency of presented method is likened to the published articles result that is tabulated in Table 1.

Table 1: Published result's parameter comparison

\begin{tabular}{ccccc}
\hline Ref & $\begin{array}{c}\mathbf{L} \\
(\mathbf{m m})\end{array}$ & $\mathbf{G b p s}$ & $\begin{array}{c}\mathbf{V} \boldsymbol{\pi} \mathbf{L} \boldsymbol{\pi} \\
(\mathbf{V . c m})\end{array}$ & $\begin{array}{c}\text { ER } \\
(\mathbf{d B})\end{array}$ \\
\hline$[19]$ & 0.76 & 40 & 1.5 & 7.02 \\
{$[20]$} & 8 & - & 3.2 & 18 \\
{$[23]$} & 1.6 & 113 & 2.4 & - \\
{$[24]$} & 5 & 100 & 2.6 & 5.6 \\
{$[25]$} & 3 & 10 & 1.09 & 11 \\
{$[26]$} & 1.3 & 100 & 0.75 & 2.4 \\
propo & 3.5 & 80 & 1.06 & 12.4 \\
sed & & & & \\
\hline
\end{tabular}

Greater ER is derived [20] through raising PS length. At [24], 5mm PS length has been utilized for getting the $\pi$ phase shift and 5.5dB ER. Diminished the length to $1.2 \mathrm{~mm}$ [27] but requires the reverse BVapproximately $6 \mathrm{~V}$, also $2.4 \mathrm{~dB}$ of ER has been acquired. Table 1 displays the PS in MZM works likened to different published outcomes.

Up to $15 \mathrm{~km}$ length of data centres span linked through fibre optic cables. The PS-MZM efficacy to communicate amid the chips coupled utilizing fibre optic cables. In $80 \mathrm{Gbps}, \lambda=$ $1552.5 \mathrm{~nm}$, the proficiency of transmission distance is assessed. Figure 9 represents the PS-MZM eye diagram and $\mathrm{V} \pi \mathrm{L}=1.05 \mathrm{~V} . \mathrm{cm} 1 \times 10^{-3}$ (among chips) that PS-MZM could transfer up to $29 \mathrm{~km}$. Figure 6 depicts RF VPP is $8 \mathrm{~V}$ including extinction ratio $>13 \mathrm{~dB}$ for necessary $\mathrm{PN}$-junction mode with linear including the direction of propagation. RF VPP is lessened to $\sim 6.5 \mathrm{~V}$ with PS in serpentine PN-junction. The reduction of voltage modulation is attained with structured modification utilizing serpentine design. It assures PS-MZM is appropriateto intra data centre utilizations alongwave transmission of guided (optical fibre) with unguided (free space optics). The transmission length is maximized utilizing amplifier.

The speed capability of PS-MZM is deemed through changing the bit rate, and then compute the BER. With maximizing bit rate, BER maximizes as inferred from figure 10. Here, PS-MZM aids to $95 \mathrm{Gbps}$. The bit rate is increased by enhancing the responsivity of PIN photo recognizers or presenting the filter. A significant lessening of radio frequency VPP lesser 3.3 VPP including extinction ratio of 11-dB for PS-MZM containing 3-mm vertical PN-junction PS. Figure 6.27 vertical PN junction profile is depicted. PS-MZM has been acquired $\sim 0.25$ and $2.7-\mathrm{V} d c \mathrm{~V} \pi$. PS-MZM at vertical PN junction is lesser then PS-MZM simulated on $-3 \mathrm{~V}$ dc reversebias voltages at serpentine mode. In modulation voltage, 
configuration of vertical PN junction contains lessening benefit.

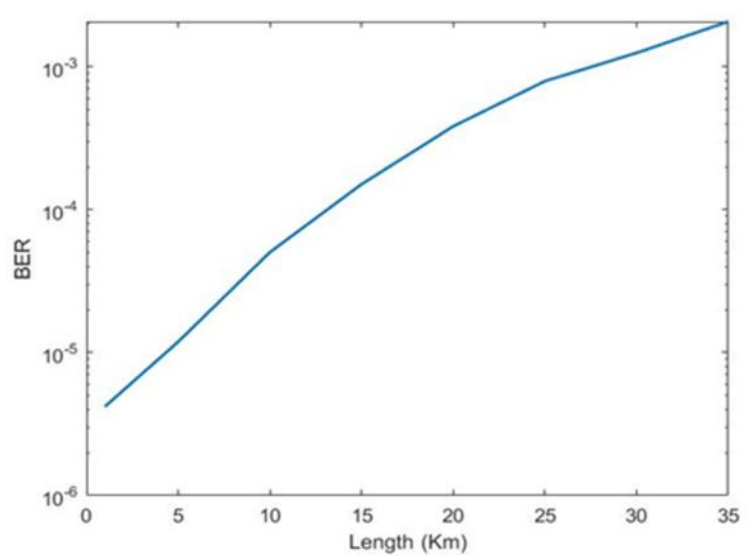

Figure 10: PS-MZM transmission capacity over 80 Gbps fibre optic cable

\section{Discussion}

The power consumption of this method is measured from currents to EIC via voltage supply. The currents drawn by $6 \mathrm{~V}$, 1.2 $\mathrm{V}$ biases are $60.09 \mathrm{~mA}, 41.82 \mathrm{~mA}$ for $44 \mathrm{Gbps}$ mode, in consequence $410 \mathrm{~mW}$ consumption of power. The power consuming is greater than the reported that is $300 \mathrm{~mW}$ in $50 \mathrm{Gbps}$ operation, because extra direct current flows from driver output via $50 \Omega$ modulator termination resistor for ground. Experimental including theoretical assessment on silicon rib-waveguide carrier-depletion PS containing certain $\mathrm{PN}$ junction architectures are expressed in light of high ME for the capacity of higher transmission energy-efficient photonic networks.

\section{CONCLUSION}

In this manuscript, the plus-shaped PN junction PS is deemed. This model is enhanced the modulation efficiency (ME) as well as decreased optical loss for higher-speed DR. To get greater efficiency of modulation, the $\mathrm{P}$ doped region width and the thickness of doped regions are differ under slabs. The aim is to create an optimum CD type plus-shaped PS including lesser V $\pi$ L. Silicon MZM including proposed PS can satisfy the higher-speed data transmission requirement on the applications of inter with intra data centre. The simulation analysis of circuit-level is executed in the proposed PS acquired at travelling wave electrode (TWE) silicon Mach Zehnder modulator (SMZM). In $80 \mathrm{Gbps}$, the $12.39 \mathrm{~dB}$ maximal extinction ratio along $8.67 \times 10-8$ bit error rate $(B E R)$ is acquired in $\mathrm{V} \pi \mathrm{L} \pi$ of $1.05 \mathrm{~V} . \mathrm{cm}$ for $3.5 \mathrm{~mm}$ PS length. The measured intrinsic bandwidth of $3 \mathrm{~dB}$ denotes $\sim 38 \mathrm{GHz}$, whereas the transmission energy per bit denotes $1.71 \mathrm{pJ} / \mathrm{bit}$. The modulator is structured for fulfilling the data centre futuristic requirements, also utilized in another utilization, viz optical switches. PS using SMZM, ER $12.39 \mathrm{~dB}$ as well as
$8.67 \times 10^{-8}$ BER acquires in $1.05 \mathrm{~V} . \mathrm{cm} \mathrm{V} \pi \mathrm{L} \pi$ for the wavelength of $1552.5 \mathrm{~nm}$. The modulator is structured for fulfilling the data centre futuristic requirements, also utilized in another utilizations, viz optical switches. The PS in MZM is examined to its associate distance $(29 \mathrm{~km}$ for inter with intra data centre interlink communication without amplifier). With the help of complex modulation modes, more enhancements in bit rate is derived. More examines are carried out to recognize the maximal communication distance using proposed PS under SMZM for the requirements of data centre. Rib-waveguide PS modulation proficiency is categorized utilizing SMZM that indicates $\pi$ phase shift Vs PS optical loss, $\pi$-shift voltage. Owing to the absorption of free-carrier, compensation doping profile of $\mathrm{PN}$-junction is thinly doped segments at core of ribwaveguide permit the minimization of optical loss, thus increasing SMZM. More development of SMZM is predicted with serpentine $\mathrm{PN}$ junction layout testing with theoretical investigations in silicon rib-waveguide carrier-depletion PS containing certain PN junction models are expressed higher $\mathrm{ME}$ for the capacity of higher-transmission energy-efficient photonic networks. At lateral, Si MZMs higher-speed function with vertical PN-junction scheme is classified at $10-\mathrm{Gb} / \mathrm{s}$ eyediagram. RF VPP is lesser 3.3 VPP including extinction ratio of 11-dB vertical PN-junction PS.

\section{REFERENCE}

[1] Perspectives and C. Report, "Cisco Annual Internet Report - Cisco Annual Internet Report (2018-2023) White Paper", Cisco, 2020. [Online]. Available: https://www.cisco.com/c/en/us/solutions/collateral/se rvice-provider/visual-networking-index-vni/whitepaper-c11-741490.html.

[2] S. Harish, X. Xu, A. Hosseini, X. Zhang, Y. Zhang, D. Kwong, and R. T. Chen. "Recent advances in silicon-based passive and active optical interconnects." Optics express 2015: 2487-2511..

[3] S. Mythili, K. Thiyagarajah, P. Rajesh, FH. Shajin. "Ideal position and size selection of unified power flow controllers (UPFCs) to upgrade the dynamic stability of systems: an antlion optimiser and invasive weed optimisation algorithm". HKIE Trans. 2020; 27(1):25-37.10.33430/V27N1THIE-2018-0024.

[4] T. David, A. Zilkie, E. John. Bowers, T. Komljenovic, T. Graham. R. Laurent V. Delphine M.-Morini "Roadmap on silicon photonics." Journal of Optics 18, no. 7 2016: 073003..

[5] Q. Xu, S. Manipatruni, B. Schmidt, J. Shakya and M. Lipson, "125 Gbit/s carrier-injection-based silicon micro-ring silicon modulators", Optics Express, vol. 15, no. 2, pp. 430, 2007.

[6] W. Green, M. Rooks, L. Sekaric and Y. Vlasov, "Ultra-compact, low RF power, $10 \mathrm{~Gb} / \mathrm{s}$ silicon 
Mach-Zehndermodulator", Optics Express, vol. 15, no. 25, pp. 17106, 2007.

[7] L. Chen, K. Preston, S. Manipatruni and M. Lipson, "Integrated $\mathrm{GHz}$ silicon photonic interconnect with micrometer-scale modulators and detectors", Optics Express, vol. 17, no. 17, pp. 15248, 2009.

[8] A. Luca, R. Palmer, S. Diebold, K. Philipp Pahl, B. Chen, R. Dinu, M.Fournier. "100 GHz siliconorganic hybrid modulator." Light: Science \& Applications 3, no. 5 2014: e173-e173..

[9] N. Youngblood, Y. Anugrah, R. Ma, S. Koester and M. Li, "Multifunctional Graphene Optical Modulator and Photodetector Integrated on Silicon Waveguides", Nano Letters, vol. 14, no. 5, pp. 27412746, 2014.

[10] X. Zhang. C. J.Chung. A. Hosseini, H. Subbaraman, J. Luo, A. Jen, K., R. T. Chen, "High performance optical modulator based on electro-optic polymer filled silicon slot photonic crystal waveguide". Journal of Lightwave Technology, 2015 34(12), 2941-2951.

[11] J. Baek, J. You and K. Yu, "Free-carrier electrorefraction modulation based on a silicon slot waveguide with ITO", Optics Express, vol. 23, no. 12, pp. 15863,2015

[12]H. Dalir, Y. Xia, Y. Wang and X. Zhang, "Athermal Broadband Graphene Optical Modulator with 35 GHz Speed", ACS Photonics, vol. 3, no. 9, pp. 15641568, 2016.

[13] S. Rajput, V. Kaushik, S. Jain and M. Kumar, "Slow Light Enhanced Phase Shifter Based on Low-Loss Silicon-ITO Hollow Waveguide", IEEE Photonics Journal, vol. 11, no. 1, pp. 1-8, 2019.

[14] S. Rajput, V. Kaushik, S. Jain, P. Tiwari, A. Srivastava and M. Kumar, "Optical Modulation in Hybrid Waveguide Based on Si-ITO Heterojunction", Journal of Lightwave Technology, vol. 38, no. 6, pp. 1365-1371, 2020.

[15]P. Rajesh, F. H. Shajin "A Multi-Objective Hybrid Algorithm for Planning Electrical Distribution System". European Journal of Electrical Engineering. 2020;22(4-5):224-509. doi:10.18280/ejee.224-509

[16]R. Soref and B. Bennett, "Electrooptical effects in silicon", IEEE Journal of Quantum Electronics, vol. 23, no. 1, pp. 123-129, 1987.

[17] A. Liu, L. Liao, D. Rubin, H. Nguyen, B. Ciftcioglu, Y. Chetrit, M. Paniccia, "High-speed optical modulation based on carrier depletion in a silicon waveguide". Optics express, 2007 15(2), 660-668..

[18] F. H. Shajin, P. Rajesh "Trusted Secure Geographic Routing Protocol: outsider attack detection in mobile ad hoc networks by adopting trusted secure geographic routing protocol". International Journal of Pervasive Computing and Communications. 2020; ahead-of-print(ahead-of-print). doi:10.1108/ijpcc-09-2020-0136

[19] M. Ziebell, D. Marris-Morini, G. Rasigade, J. M Fédéli, P. Crozat, E. Cassan, L. Vivien,. "40 Gbit/s low-loss silicon optical modulator based on a pipin diode". Optics express, (2012)20(10), 10591-10596..

[20] R. JesuwanthSugesh and A. Sivasubramanian, "Redesigning Mach-Zehnder Modulator with Ring Resonators", Lecture Notes in Electrical Engineering, pp. 185-191, 2017.

[21] P. Dong, L. Chen and Y. Chen, "High-speed lowvoltage single-drive push-pull silicon Mach-Zehnder modulators", Optics Express, vol. 20, no. 6, pp. 6163, 2012.

[22] H. Xu, X. Xiao, X. Li, Y. Hu, Z. Li, T. Chu, J. Yu,. "High speed silicon Mach-Zehnder modulator based on interleaved PN junctions". Optics express, 2012 20(14), 15093-15099..

[23] A. Rao, A. Patil, P. Rabiei, A. Honardoost, R. DeSalvo, , A. Paolella, S. Fathpour. "Highperformance and linear thin-film lithium niobate Mach-Zehnder modulators on silicon up to 50 GHz". Optics letters, 2016 4l(24), 5700-5703.

[24] M. Félix Rosa, L. Rathgeber, R. Elster, N.Hoppe, T. Föhn, , M. Schmidt, M. Berroth. "Design of a carrierdepletion Mach-Zehnder modulator in $250 \mathrm{~nm}$ silicon-on-insulator technology". Advances in Radio Science, 2017. 15, 269-281.

[25]T. Ang, C. Png and S. Lim, "Numerical analysis and optimization of high-speed silicon microring resonator modulators utilizing higher-performance carrier-depletion phase shifters", Silicon Photonics XII, 2017

[26] MK . Thota, FH . Shajin, P. Rajesh "Survey on software defect prediction techniques. International Journal of Applied Science and Engineering", 2020;17;331-344. journal of electrical engineering-

[27] G. de Farias, Y. Bustamante, H. de Andrade, U. Moura, A. Freitas and D. de A. Motta, "Demonstration of $>48 \mathrm{GHz}$ Single-Drive Push-Pull SMZM with Lower $\mathrm{V}<\inf >\Pi</ \inf >$ L", 2018 IEEE 15th International Conference on Group IV Photonics (GFP), Cancun, Mexico, 2018, pp 1-2.

[28] M. He, M. Xu, Y. Ren, J. Jian, Z. Ruan, Y. Xu, X. Cai, "High-performance hybrid silicon and lithium niobate Mach-Zehnder modulators for 100 Gbit s- 1 and beyond". 2019. Nature Photonics, 13(5), 359364.

[29] K. Ogawa, K. Goi, N. Ishikura, H. Ishihara, S. Sakamoto, T. Y. Liow, C. E. Png, "Silicon-based 
phase shifters for high figure of merit in optical modulation". In Silicon Photonics XI 2016 (. International Society for Optics and Photonics.

[30] R. Palmer, L. Alloatti, D. Korn, P. C. Schindler, R. Schmogrow, W. Heni, J. Leuthold,. Silicon-organic hybrid MZI modulator generating OOK, BPSK and 8-ASK signals for up to $84 \mathrm{Gbit} / \mathrm{s}$. 2013. IEEE Photonics Journal, 5(2), 6600907-6600907.

[31] S. Jain, S. Rajput, V. Kaushik and M. Kumar, "High speed optical modulator based on silicon slotted-rib waveguide", Optics Communications, vol. 434, pp. 49-53, 2019

[32] S. Jain, S. Rajput, V. Kaushik, Sulabh and M. Kumar, "Efficient Optical Modulation With High Data-Rate in Silicon Based Laterally Split Vertical p-n Junction", IEEE Journal of Quantum Electronics, vol. 56, no. 2, pp. 1-7, 2020.

[33] T. Baehr-Jones, R. Ding, Y. Liu, A. Ayazi, T. Pinguet, N. C. Harris, M. Hochberg,. "Ultralow drive voltage silicon traveling-wave modulator". Optics express, 2012. 20(11), 12014-12020.

[34] J. Dakin and R. Brown, "Handbook of Optoelectronics (Two-Volume Set)", CRC press, 2006

[35] L. Binh, Optical multi-bound solitons, 1st ed. 2015.

[36] S. Vetova, "Workflow Model for Big Data Analysis and Visualization, Int. J. of Applied Mathematics", Computational Science and Systems Engineering, Vo. 3, pp. 99-106, 2021.

[37] A. Mohamed, "Multidimensional Data Indexing in BIG DATA, Int. J. of Applied Mathematics", Computational Science and Systems Engineering, Vol. 2, pp. 12-18, 2020.

[38] Z. Yong, S. Shopov, J.C. Mikkelsen, R. Mallard, J.C. Mak, S.P. Voinigescu, and J.K. Poon, "Flip-chip integrated silicon Mach-Zehnder modulator with a $28 \mathrm{~nm}$ fully depleted silicon-on-insulator CMOS driver". Optics express, vo. 25, no.6, pp.6112-6121, 2017.

[39] Y. Zhou, L. Zhou, M. Wang, Y. Xia, Y., Zhong, X. $\mathrm{Li}$, and J. Chen, Linearity characterization of a dualparallel silicon Mach-Zehnder modulator. IEEE Photonics Journal, vol.8,no.6, pp.1-8, 2016.

[40] A. Samani, V. Veerasubramanian, E. El-Fiky, D. Patel, and D.V. Plant, A silicon photonic PAM-4 modulator based on dual-parallel Mach-Zehnder interferometers. IEEE Photonics Journal, vol. 8, no.1, pp.1-10, 2015.

[41]E. Temporiti, A. Ghilioni, G. Minoia, P. Orlandi, M. Repossi, D. Baldi, and F. Svelto, Insights into silicon photonics Mach-Zehnder-based optical transmitter architectures. IEEE Journal of Solid-State Circuits, vol. 51, no. 12, pp.3178-3191, 2016.

[42]D. Perez-Galacho, C. Baudot, T. Hirtzlin, S. Messaoudène, N. Vulliet, P. Crozat, F. Boeuf, L. Vivien, and D. Marris-Morini, Low voltage $25 \mathrm{Gbps}$ silicon Mach-Zehnder modulator in the Oband. Optics express, vol. 25, no. 10, pp.1121711222, 2017.

[43] J. Ding, S. Shao, L. Zhang, X. Fu, and L. Yang, Method to improve the linearity of the silicon MachZehnder optical modulator by doping control. Optics express, vol. 24, no.21, pp.24641-24648, 2016.

Jesuwanth Sugesh R G (Jesuwanth Sugesh Ramesh Gabriel) has a Bachelors degree in Electronics and Communication Engineering from Karunya Institute of Science and Technology (2012), Masters degree in Communication Systems from Anna University (2016) and is currently pursuing his Ph.D. degree specializing in Silicon Photonics from Vellore Institute of Technology, Chennai. His current research topic is on analyzing and improving the efficiency of CMOS compactable silicon-based Mach Zehnder modulator for data centre application and emerging trends of silicon photonics in optical communication. He has two years of professional experience as a Software Engineering Analyst in Accenture, India. His other research areas of interest include optical communication, optical networks, bio-optical Engineering and Optical Network Management. He has published few journal papers in optical network management during his post-graduate and has participated in many international conferences. He is a member of the International Association of Engineers (IAENG), IEEE and Optical Society of India (OSI)..

A. Sivasubramanian (Arunagiri Sivasubramanian) has received B.E. degree in Electronics and Communication Engineering from the University of Madras in 1990, and M.E. in Applied Electronics from Bharathiar University in 1995 and $\mathrm{Ph} . \mathrm{D}$. degree in Optical Communication from Anna University Chennai in 2008. Currently, he is a professor of Electronics Engineering in Vellore Institute of Technology (Chennai Campus), Chennai. He has more than 20 years of experience in teaching and guiding projects for undergraduate, postgraduate and research scholars. His areas of interests include optical communication, optical networks, bio-optical Engineering, Wireless sensor, silicon photonics and computer networks. He is a member of ISTE, IETE, IEEE, and OSA.

\section{Contribution of individual authors to the creation of a scientific article (ghostwriting policy)}

Mr. R G Jesuwanth Sugesh: Conceptualization, Methodology, Writing- Original draft preparation

Dr. A Sivasubramanian: Supervision, Writing- Reviewing and Editing 


\section{Creative Commons Attribution License 4.0} (Attribution 4.0 International, CC BY 4.0)

This article is published under the terms of the Creative Commons Attribution License 4.0

https://creativecommons.org/licenses/by/4.0/deed.en_US 